\title{
Rectification Correlation between Water and Ions through Asymmetric Graphene Channels
}

Shuang Li, ${ }^{1}$ Yunzhen Zhao, ${ }^{1}$ Xinke Zhang, ${ }^{1}$ Chuxuan Ding, ${ }^{1}$ Jiaye Su ${ }^{*}, 1,2$

${ }^{1}$ Department of Applied Physics, and ${ }^{2}$ MIIT Key Laboratory of Semiconductor

Microstructure and Quantum Sensing, Nanjing University of Science and Technology, Nanjing, Jiangsu 210094, China

*Electronic mail: jysu@iccas.ac.cn; jysu@njust.edu.cn

\section{$\underline{\text { Supporting Information }}$}




\section{The flux and rectification ratio of $\mathrm{Li}^{+}, \mathrm{Na}^{+}$and $\mathrm{K}^{+}$}

In an effort to further elucidate the ion size effect, in this part, we compare the transport of three different salt solutions, i.e, $\mathrm{LiCl}, \mathrm{NaCl}$ and $\mathrm{KCl}$. Figure $\mathrm{S} 1$ a shows that the flux of three cations increases linearly with the increase of electric field E. Clearly, the cation flux has an order of $\mathrm{K}^{+}>\mathrm{Na}^{+} \geq \mathrm{Li}^{+}$, agreeing with the previous work for symmetric graphene channels [S1]. This order is same as the order for cation size and bulk diffusion coefficient [S2]. This is because the larger diameter cation has smaller hydration binding energy [S3], and should be easier to enter the channel, leading to a higher flux. The asymmetry of cation fluxes in positive and negative electric fields exist in all three solution systems. This is reasonable as the larger entrance at base facilitates the collection of ions from the bulk solution, which leads to a higher flux. Interestingly, as we can further see in Figure S1b, the rectification ratio has an opposite order of $\mathrm{Li}^{+}>\mathrm{Na}^{+} \geq \mathrm{K}^{+}$, where the values of $\mathrm{Li}^{+}$are obviously higher especially at small field strengths. This is because the negative fluxes of $\mathrm{Li}^{+}$at small E are clearly smaller than the positive ones. These results have great implications for the separation of the three cations. The cation flux can help to select $\mathrm{K}^{+}$from $\mathrm{Li}^{+}$and $\mathrm{Na}^{+}$, similar to previous symmetric graphene channels [S1]; while the rectification ratio can select $\mathrm{Li}^{+}$from $\mathrm{K}^{+}$and $\mathrm{Na}^{+}$. Consequently, the present asymmetric graphene channel provides an effective way to separate $\mathrm{Li}^{+}, \mathrm{K}^{+}$and $\mathrm{Na}^{+}$.

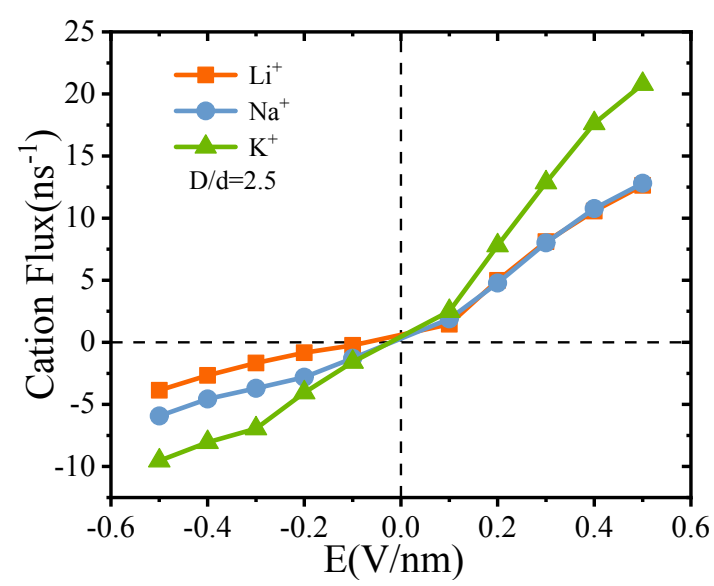

(a)

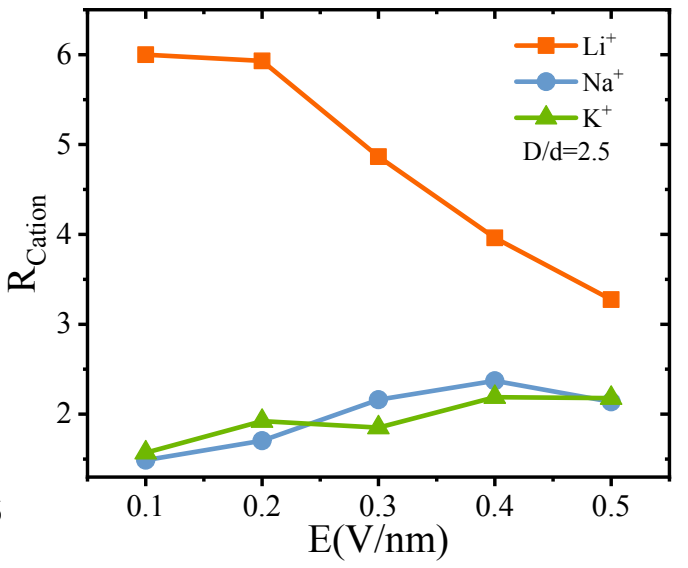

(b)

Figure S1. (a) The flux and (b) rectification ratio of cations as a function of the electric field $\mathrm{E}$ for the channel opening ratio $\mathrm{D} / \mathrm{d}=2.5$. 


\section{The translocation time of $\mathrm{Li}^{+}, \mathrm{Na}^{+}$and $\mathrm{K}^{+}$}

Figure S2 shows the translocation time of cations as a function of the field strength. Clearly, the ion translocation time decreases with increasing the field strength and follows the one-dimensional Langevin equation, similar to Figure 5 in our main text. A closer comparison indicates that the ion translocation time has an order of $\mathrm{Li}^{+}>\mathrm{Na}^{+}>\mathrm{K}^{+}$in both transport directions, in an opposite to the order of ion flux in Figure S1a. This is reasonable as short translocation time should correspond to large flux. The ion translocation time for $+\mathrm{E}$ is larger than $-\mathrm{E}$. This is because ions have to dehydrate continuously in the positive direction and are thus subjected to high friction. Although, ions are difficult to enter the tip, once they enter it they can transport out of the base easily, leading to small translocation times.

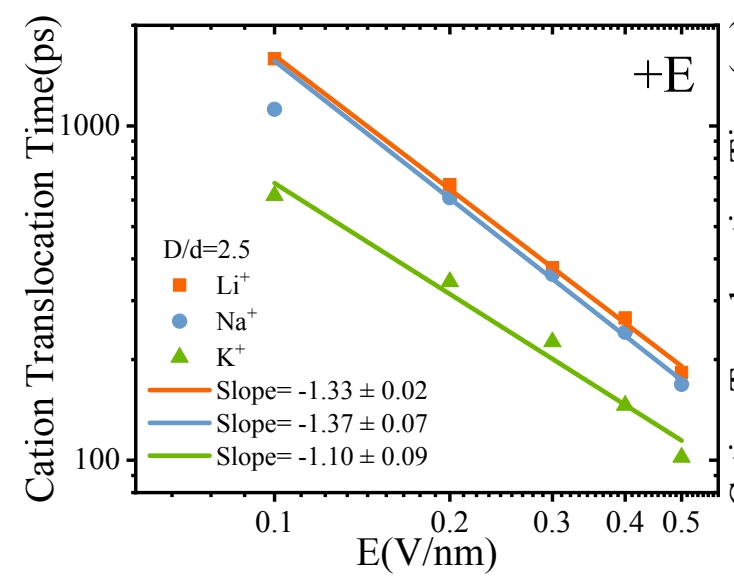

(a)

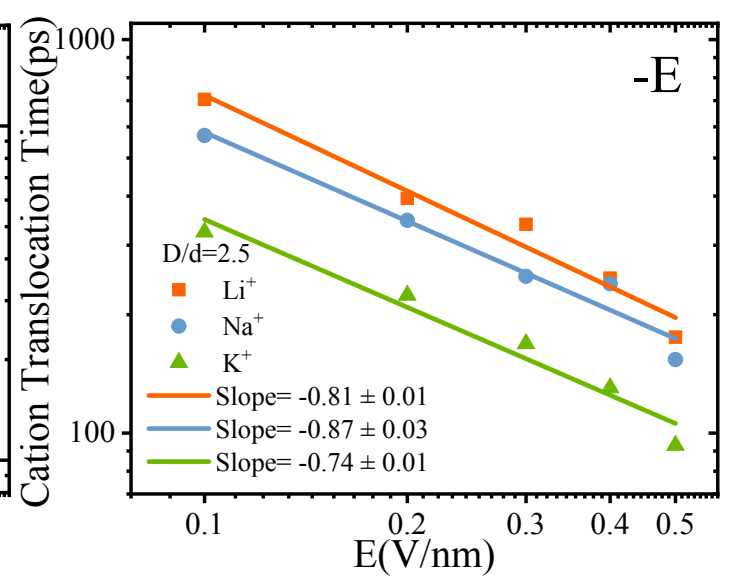

(b)

Figure S2. Power law plots of ion translocation time for the (a) positive and (b) negative electric field.

\section{The flux and rectification ratio of $\mathrm{Cl}^{-}$for the $\mathrm{LiCl}, \mathrm{NaCl}$ and $\mathrm{KCl}$ systems}

Figure S3a shows the flux of $\mathrm{Cl}^{-}$for the three salt solutions as a function of the electric field. For the negative electric field, the $\mathrm{Cl}^{-}$flux increases linearly with the increase of field strength and is almost independent of the salt types. The $\mathrm{Cl}^{-}$flux in + E weakly depends on the salt types and deviates from the linear increasing behavior. The $\mathrm{Cl}^{-}$flux for $\mathrm{NaCl}$ system is slightly smaller, leading to its higher rectification ratio in Figure S3b. 


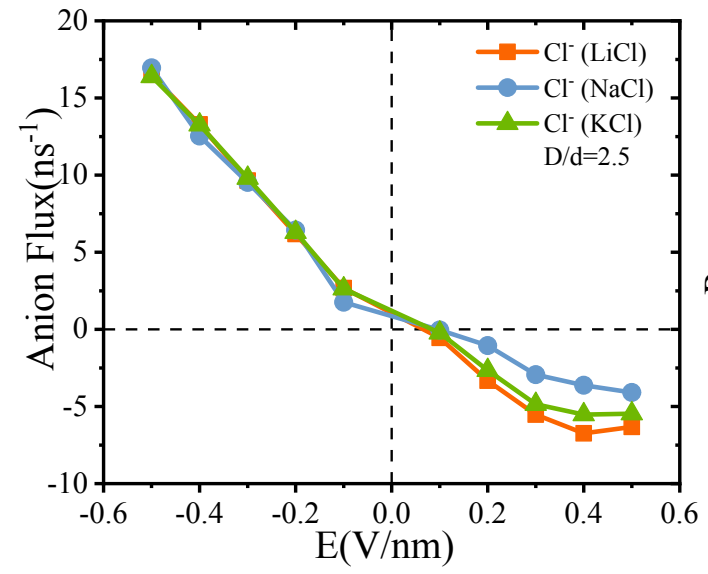

(a)

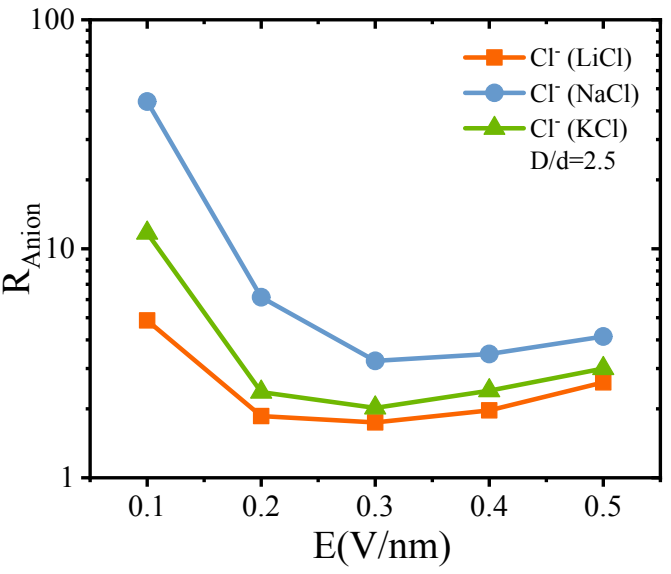

(b)

Figure S3. The (a) flux and (b) rectification ratio of $\mathrm{Cl}^{-}$as a function of the electric field $\mathrm{E}$ for $\mathrm{D} / \mathrm{d}=2.5$.

\section{The translocation time of $\mathrm{Cl}^{-}$for the $\mathrm{LiCl}, \mathrm{NaCl}$ and $\mathrm{KCl}$ systems}

It can be seen in Figure S4 that the translocation time of $\mathrm{Cl}^{-}$also has a power law relation with $\mathrm{E}$, where the slopes and values slightly depend on the salt types, similar to the above cation behaviors.

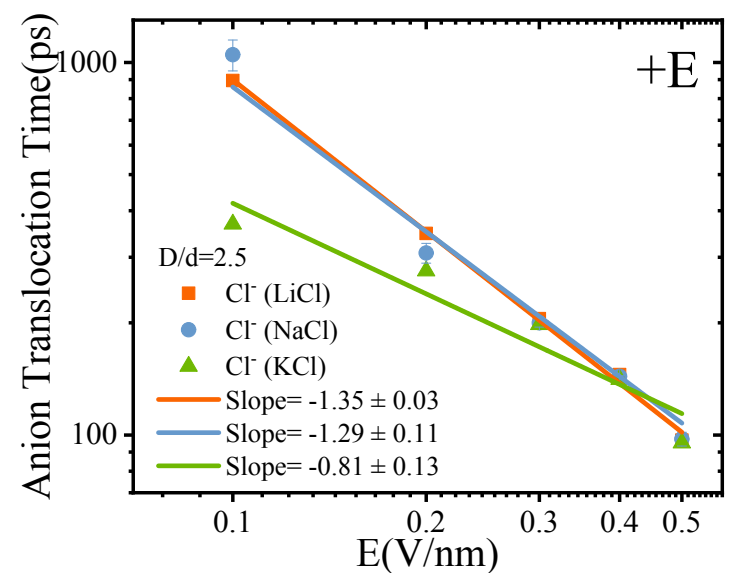

(a)

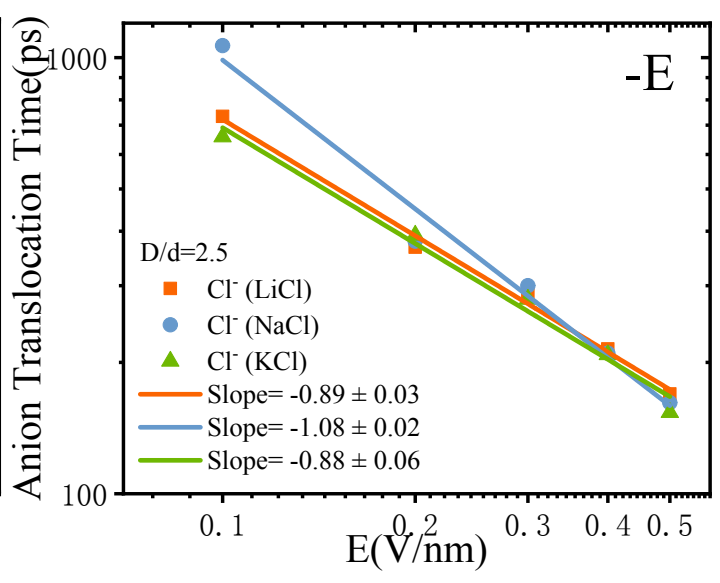

(b)

Figure S4. Power law plots of $\mathrm{Cl}^{-}$translocation time for the (a) positive and (b) negative electric field.

\section{REFERENCES}

[S1] Y. Z. Zhao, D. C. Huang, J. Y. Su, and S. W. Gao. Coupled transport of water and ions through graphene nanochannels. J. Phys. Chem. C 2020, 124, 17320-17330. 
[S2] S. Chowdhuri, and A. Chandra, Hydration structure and diffusion of ions in supercooled water: ion size effects. J. Chem. Phys. 2003, 118, 9719-9725.

[S3] Zhou, J.; Lu, X.; Wang, Y.; Shi, J. Molecular Dynamics Study on Ionic Hydration. Fluid Phase Equilib. 2002, 194-197, 257-270. 